\title{
Performance Comparison of Artificial Intelligence Approaches for Battery Energy Storage Size Optimization in PV Micro-grid
}

\author{
T. Kerdphol, K. Fuji, Y. Mitani, and Y. Qudaih
}

\begin{abstract}
Studies into the determining size of battery energy storage system (BESS) has become significant recently, owing to their use in a variety of complex, high performance and energy storage system applications. This paper presents a comparative study of optimization techniques between particle swarm optimization (PSO) and artificial neural network (ANN) for evaluating the optimum size of BESS in the micro-grid system. In this paper, the micro-grid system consists of two micro turbine systems, solar photovoltaic (PV) system and BESS, and it is connected to the utility grid. Simulation results show that the optimal size of BESS-based PSO approach achieves the lowest performance in achieving the optimal BESS size compared to the optimal size of BESS-based ANN. However, the optimal sizing of BESS-based ANN approach gives the fastest conversion time compared to the optimal sizing of BESS-based PSO.
\end{abstract}

Index Terms-Artificial neural network (ANN), battery energy storage system, frequency control, micro-grid, particle swarm optimization.

\section{INTRODUCTION}

One of the most important providers of rustic electrification projects demonstrates that almost 1.2 billion people do not have regular access to electricity. They live with difficulties that mostly are in rural areas. Micro-grid is considered to as a local grid incorporating with decentralized or distributed generations (DGs), battery energy storage system (BESS), and local loads, can operate in either in grid-connected mode or isolated mode, with feasibility of seamless transitions between them [1]. In isolated mode, the power transfer among DGs, BESS, and local loads should be balanced inside the stand-alone micro-grid in order to maintain the frequency stable. The advantages are found to be in power generation flexibility, security, reliability, and also improved power quality. As micro-grids do not send power over vast distances, so, they can make better use of low to medium voltage systems requiring less metal involved in heavy lines and switching equipment to accommodate loads for decreasing pollution issue and resource consumption. Another advantage is that it is feasibility to have dissimilar types of generation. For example, a micro-grid can use solar or wind to obtain the advantages of both and improve generating ability [2]. However, as the output characteristics

Manuscript received May 18, 2015; revised October 12, 2015.

T. Kerdphol, K. Fuji, Y. Qudaih, and Y. Mitani are with the Kyushu Institute of Technology, Kitakyushu, Japan (e-mail: thongchartkerd@gmail.com, fuji19690708@gmail.com, yaser_qudaih@yahoo.com,mitani@ele.kyutech.ac.jp). of DGs in a micro-grid are quite different from the conventional energy sources and the output of DGs cannot predict, a micro-grid should be capable of handling unexpected fluctuation and maintaining system reliability. Hence, BESS plays an important role for obtaining the purpose of power balance and grid frequency support in the micro-grid [3].

BESS can be used in various aspects of power system as one key factor for sustainable energy in many countries. Typical distribution uses of BESS are peak load management, stabilizing energy production from intermittent generations to enhance distributed energy resources integration, and dealing with emergency circumstances such as blackout, particular in micro-grids. BESS is a technology which may become a solution for distribution applications if costs reduce and regulators allow these kinds of uses. In many countries such as France, it has been presented that BESS be implemented in three tiers. Two of these, at the residential and low volt grid levels, will facilitate the injection of large amounts of distributed solar PV energy into the distribution grid. A third tier of BESS will support in the area of reliability.

At the residential level, BESS will be installed in the homes of clients who take part in the illustration and have installed solar PV panels on their roofs. Each BESS will be located at the client's electrical panel, while the solar PV equipment will be located at the grid. A dedicated platform using asymmetric digital subscriber line infrastructure will control the operation of these residential BESS units and power usage of individual clients. Every two minutes, the smart meter will send client power consumption and generation data to the control box in the actual time [4].

In the case of small disturbance, BESS is discharging when the system frequency is lower than 50 or $60 \mathrm{~Hz}$. On the other hand, BESS is charging when the system frequency is higher than 50 or $60 \mathrm{~Hz}$. In the case of large disturbance, BESS can enhance the performance of the system frequency control by integrating BESS with under frequency load shedding scheme, or under frequency generation trip, and over frequency generation trip. With these different functions, BESS can offer a good solution. It can be concluded that BESS is a rapid and flexible element for power system [5]-[8]. Moreover, the purpose of optimal BESS is to smoothen the power in a system with wind or solar energy. In such a system, BESS can play a role by absorbing the surplus power and compensating the power shortage due to the uncertainties of renewable energy.

The optimal size of BESS is an important aspect to maximize the benefits of BESS in the micro-grid. The 
inappropriate size of BESS can cause low or over frequency and voltage to the micro-grid system [9], [10]. Moreover, there is no clearly suggestion for choosing the optimal location of BESS in a micro-grid system [11]. Hence, it is essential to consider an appropriate size and location of BESS to the micro-grid system in order to improve system performance, power quality and reliability.

There are many optimization techniques which can be used for determining the optimal BESS size. The optimization method can be achieved by means of linear programming method, enumerative method, iterative algorithm, Artificial Neural Network (ANN) [12]-[16], particle swarm optimization (PSO) and so on [17]-[20].

This study aims at comparing the performance of artificial intelligence approaches for determining the BESS size in the micro-grid system. PSO and ANN are selected to be a candidate for comparison in terms of accurate performance and time of calculation. This remain of this paper is organized as follow. The micro-grid system that studied and implemented is introduced in Section II. BESS size optimization approaches are briefly described in Section III. The simulation results and analysis are shown in Section IV. Finally, Section V concludes the work.

\section{PROCEDURE FOR PAPER SUBMISSION}

\section{A. Architecture of Micro-grid}

A Micro-grid, depicted as a subset of the grid which can be isolated (e.g., at the level of a college or campus), is able to provide all/part of a customer's load during an outage or in case of grid emergencies. The customer may not beck-feed into the grid or supply third-party loads during outage conditions, due to safety and liability concerns. A typical characteristic of a micro-grid is that it can be operated either in grid-connected mode or stand-alone mode. Under normal operation, the micro-grid is connected to the utility grid.

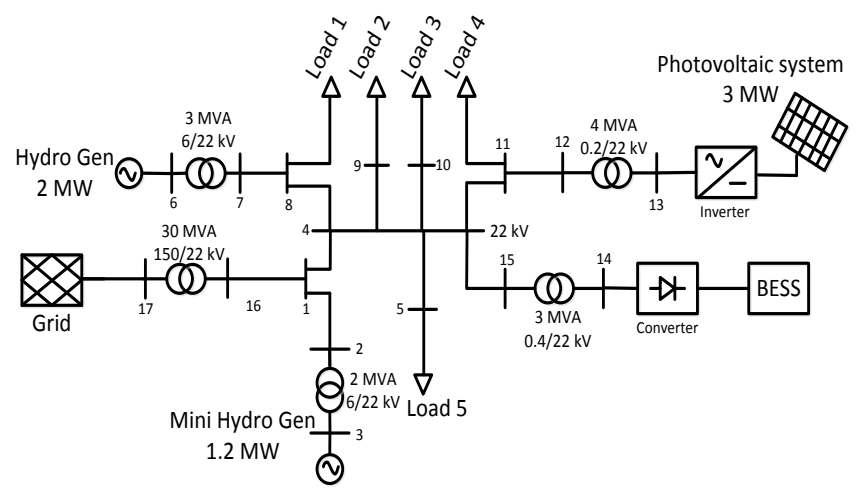

Fig. 1. Micro-grid structure.

Fig. 1 shows the proposed micro-grid system which consists of a 1.2 MW mini-hydro generator, $2 \mathrm{MW}$ hydro generator and $3 \mathrm{MW}$ solar PV. BESS is connected to the micro-grid system at bus 14. Each DG unit has its own local controller to handle the relevant electrical variables. The system also consists of group of feeders which could be part of the distribution system. The critical load 1 with the peak power $1.85 \mathrm{MW}$ and the critical load 4 with the peak power $1.90 \mathrm{MW}$ require a local generation and the non-critical load
2, 3, 5 with the peak power 1.70 MW, 1.75 MW, 2.40 MW respectively are not connected to any local generation.

\section{B. Solar Photovoltaic Generation}

The output power of solar photovoltaic (PV) generation is uncertain as it is mostly affected by the environmental factors, namely the environmental random changes will inevitably lead to constantly changing of output power of solar PV [21]. In order to represent solar PV characteristic in operating conditions, the influences of solar insolation and ambient temperature are designed. The temperature effect is represented by a temperature coefficient of power $T_{c o}\left(1 / \mathrm{c}^{\circ}\right)$. The inverter efficiency is multiplied by the DC output convert DC to AC output as shown in (1).

$$
P_{P V}=n_{P V} P_{\text {rate } P V}\left(G / G_{0}\right)\left(1-T_{c o}\left(T_{A}-25^{\circ}\right)\right) \eta_{i n v} \eta_{\text {rel }} \text { (1) }
$$

where $n_{P V}$ is the number of solar PV modules, $P_{\text {rate } P V}$ is the rated $\mathrm{PV}$ array electrical power $(\mathrm{W}), G$ is the global insolation on the solar PV array $\left(\mathrm{W} / \mathrm{m}^{2}\right), G_{0}$ is the standard amount of insolation used to rate the capacity of solar PV modules $\left(\mathrm{W} / \mathrm{m}^{2}\right), T_{A}$ is the ambient temperature, $T_{C O}$ is the temperature coefficient of the maximum power of solar PV, $\eta_{\text {rel }}$ is the relative efficiency of the solar PV modules, $\eta_{i n v}$ is the efficiency of the inverter.

\section{Battery Energy Storage System (BESS)}

BESS can be utilized in several implementations such as peak shaving, real power control and load leveling. This paper uses BESS for enhancing the performance of frequency control as BESS can provide active power compensation in a short period of time. Recently, a huge number of electric companies and independent system operators have shown growing interest in BESS due to decreasing cost of batteries. With the fast development of technologies, BESS is expected to be used in several applications including the one proposed in this paper.

In this study, BESS operation during outage conditions is strictly limited to supply only loads on the customer's side, not back-feed power into the grid. In fact, when the power generated by the micro-grid system is greater than the load demand, the surplus power can be stored in BESS for future use. On the other hand, when there is any deficiency in the power generation of the micro-grid, the stored power can be used to supply the load. Thus, this will improve the system performance and reliability.

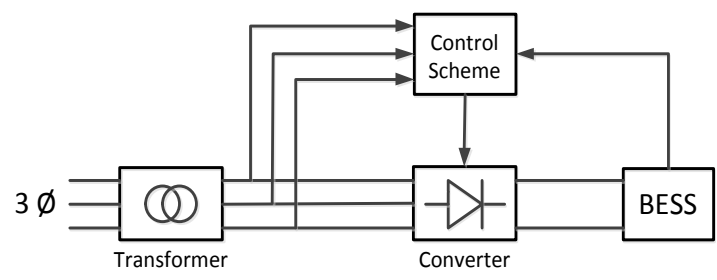

Fig. 2. BESS structure.

where, $\alpha_{i}$ is the firing delay angle of converter $i, E_{d 0}$ is the maximum DC voltage of the batteries, $E_{b 1}$ is the battery overvoltage, $E_{b t}$ is the terminal of equivalent battery, $E_{b o c}$ is the battery open circuit voltage, $I_{B E S S}$ is the DC current though the battery, $P_{B E S S}$ is the active power provided by the batteries, 
$r_{b t}$ is the connecting resistance, $r_{b s}$ is the battery internal resistance, $r_{b p}$ is the self discharge resistance, $r_{b 1}$ is the overvoltage resistance, $\Delta f$ is the frequency deviation, $X_{c o}$ is the commutating reactance, $\Delta E_{c o}$ is the DC voltage without overlap, $K_{b}$ is the control loop gain, $T_{b}$ is the measurement device time constant.

The structure of BESS consists of power converters, battery cells and control parts [6], which are shown in Fig. 2. From the schematic structure of BESS, the output of DC voltage is shown as:

$$
\Delta E_{d o}=\frac{6 \sqrt{6}}{\pi} E_{t}
$$

where $E_{t}$ is AC voltage between the line to neutral.

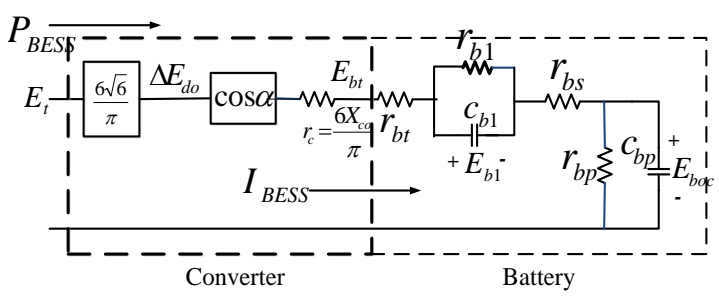

Fig. 3. Equivalent circuit.

The equivalent circuit of BESS consists of a converter connected to an equivalent battery as shown in Fig. 3. The terminal voltage of the equivalent battery can be calculated from:

$$
\begin{gathered}
E_{b t}=\Delta E_{d o} \cos \alpha-r_{c} I_{B E S S} \\
=\frac{3 \sqrt{6}}{\pi} E_{t}\left(\cos \alpha_{1}-\cos \alpha_{2}\right)-\frac{6}{\pi} X_{c o} I_{B E S S}
\end{gathered}
$$

According to the equivalent circuit of BESS, the expression of DC current flowing into the battery can be expressed as:

$$
I_{B E S S}=\frac{E_{b t}-E_{b o c}-E_{b 1}}{r_{b t}+r_{b s}}
$$

where

$$
\begin{gathered}
E_{b o c}=\frac{r_{b p}}{1+S T_{b p}} I_{B E S S} \\
E_{b 1}=\frac{r_{b 1}}{1+S T_{b 1}} I_{B E S S} \\
T_{b p}=r_{b p} C_{b p} \\
T_{b 1}=r_{b 1} C_{b 1}
\end{gathered}
$$

From the converter circuit analysis, the active and reactive powers absorbed by BESS are:

$$
P_{B E S S}=\frac{3 \sqrt{6}}{\pi} I_{B E S S} E_{t}\left(\cos \alpha_{1}+\cos \alpha_{2}\right)
$$

$$
Q_{B E S S}=\frac{3 \sqrt{6}}{\pi} I_{B E S S} E_{t}\left(\sin \alpha_{1}+\sin \alpha_{2}\right)
$$

The incremental active power is considered in load frequency control. So, $P$-modulation strategy is presented to this paper. For $P$-modulation, $\alpha_{1}=-\alpha_{2}=\alpha$. Thus;

$$
\begin{gathered}
P_{B E S S}=\frac{6 \sqrt{6}}{\pi} I_{B E S S} E_{t} \cos \alpha \\
Q_{B E S S}=0 \\
\Delta P_{B E S S}=I_{B E S S}^{0} \Delta E_{d}
\end{gathered}
$$

After that, the use of BESS in load frequency control is touched by a damping signal $\Delta E_{d}$ :

$$
\Delta E_{d}=\frac{K_{b}}{1+S T_{b}} \Delta f
$$

The $\Delta f$ is the beneficial feedback from the power system in order to provide a damping effect. Linearizing equations (1) to (13), the block diagram of BESS can be shown in Fig. 4.

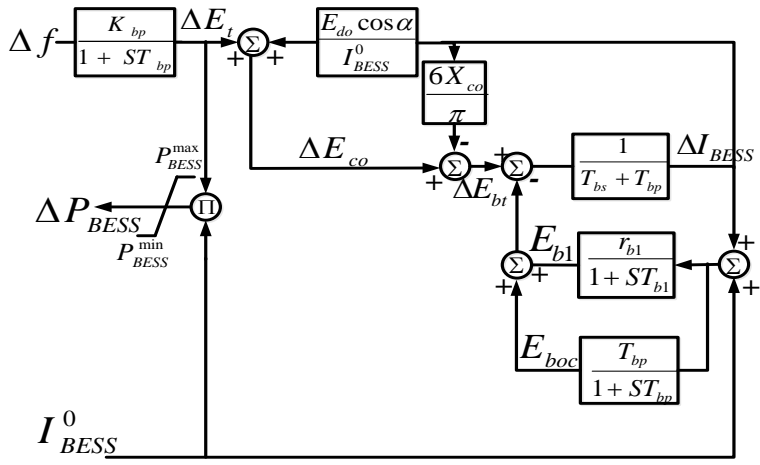

Fig. 4. Linearized BESS model for LFC.

\section{BESS SIZE OPTIMIZATION}

\section{A. Optimal BESS Size Based-PSO Approach}

Particle swarm optimization is an algorithm technique for searching optimal parameters of complicated search spaces. PSO is initiated with a group of random particles to find for optimal parameters by updating generations. In each iteration, each particle is updated by two values. These values are called $P_{\text {best }}$ and $G_{\text {best }}$ respectively. $P_{\text {best }}$ is the best solution acquired by each particle itself in all of the previous generations. $G_{\text {best }}$ is the best value obtained by any particle in all previous iterations. This value is called the best global solution. Each particle updates its position and velocity by using (15) and (16).

$$
\begin{gathered}
v_{i+1}=v_{i}+c_{1} r_{1}\left(P_{\text {best }}-x_{i}\right)+c_{2} r_{2}\left(G_{\text {best }}-x_{i}\right) \\
x_{i+1}=v_{i+1}+x_{i}
\end{gathered}
$$

where $i^{\text {th }}$ is the iteration number, $j^{\text {th }}$ is the particle number, $v_{i}$ is the velocity of a particle at iteration $i^{\text {th }}, x_{i}$ is the position of a 
particle at iteration $i^{\text {th }}, P_{\text {best }}$ is the best solution at iteration $i^{\text {th }}$, $G_{\text {best }}$ is the best global solution at iteration $i^{\text {th }}, r_{1}$ is the random number one between 0 and $1, r_{2}$ is the random number two between 0 and 1 and $c_{1}, c_{2}$ are the learning factors

The learning factors have important effects on the algorithm convergence rate. Further information for PSO can be found in [17]-[20]. In this paper, the number of particles in swarm $\left(N_{P}\right)$ is 20 . The number of iterations $\left(N_{I}\right)$ is 30 . Learning factors are $C_{1}$ and $C_{2}$ which equal to 1.49445 . The inertia weight $(w)$ is 0.7920 .

In this approach, the purpose of objective functions is to improve the frequency control of BESS in the micro-grid by integrating optimal size of BESS. To minimize the power of BESS, the final objective function is chosen and expressed as:

$$
\text { Minimize } f_{1}=\lambda J_{1}
$$

where $J_{1}$ is the objective function representing the power capacity of BESS and $\lambda$ is the weight associated to this function.

Some of these that needs to satisfied are follows:

$$
P_{B E S S}^{\min } \leq P_{B E S S}(t) \leq P_{B E S S}^{\max }
$$

where $P_{B E S S}(t)$ is the rated power capacity of BESS, $P_{B E S S}^{\min }$ is the allowed minimum rated power capacity and $P_{B E S S}^{\max }$ is the allowed maximum rated power capacity of BESS

$$
C_{B E S S}^{\min } \leq C_{B E S S}(t) \leq C_{B E S S}^{\max }
$$

where $C_{B E S S}(t)$ is the rated energy capacity of BESS, $C_{B E S S}^{\min }$ is the allowed minimum rated energy capacity and $C_{B E S S}^{\max }$ is the allowed maximum rated energy capacity of BESS.

$$
F_{\min } \leq F(t) \leq F_{\max }
$$

where $F(t)$ is the nominal frequency of the micro-grid, $F_{\min }$ is the allowed minimum nominal frequency of the micro-grid and $F_{\max }$ is the allowed maximum nominal frequency of the micro-grid.

$$
V_{\min } \leq V(t) \leq V_{\max }
$$

where $V(t)$ is the nominal voltage of the micro-grid, $V_{\min }$ is the allowed minimum nominal voltage of the micro-grid and $V_{\max }$ is the allowed maximum nominal voltage of the micro-grid.

Based on Fig. 5, the outline of the whole process of the proposed PSO-based optimal size of BESS is shown.

\section{B. Optimal BESS Size Based-ANN Approach}

Multilayer perceptron, trained by the standard back-propagation (BP) leaning algorithm, is known as the feed-forward back-propagation neural network. In this paper, the multilayer perceptron consists of three layer neurons. It shows that one hidden layer is contained and one type of activation function is used in the hidden layer. Hence, this paper can find the optimal size of BESS to supply the micro-grid when the micro-grid is separated from the main grid based on the measured voltage and frequency of the micro-grid [12]-[16]. As a nonlinear modelling method, the multilayer perceptron neural network has nonlinearity quality and it can guarantee the accuracy of fitting and there are two inputs with one output in this study as shown in Fig. 6.

where $F$ is the nominal frequency of the micro-grid system (Hz.) while $V$ is the nominal voltage of the micro-grid system (pu). $P_{B E S S}$ means the rated power capacity of BESS (MW).

The output value of the neural network is initiated by transforming a weight total of input based on a transfer function. The transfer function is used to convert the activate level of a neuron to an output value. In this paper, the hyperbolic tangent transfer function (Tanh) is activated between the input layer and the output layer as follow:

$$
y_{i}=f_{\text {Tanh }}\left(x_{i}\right)=\frac{e^{x}-e^{-x}}{e^{x}+e^{-x}}
$$

The hyperbolic tangent activation function is categorized in the logistic type and the output value saturated between -1 to +1 .

First, the output of the hidden layer can be shown as:

$$
\left[\begin{array}{c}
g_{1} \\
g_{2} \\
\bullet \\
g_{i}
\end{array}\right]=f_{\text {Tanh }}\left(\left[\begin{array}{cc}
W_{11}^{(1)} & W_{21}^{(1)} \\
W_{12}^{(1)} & W_{22}^{(1)} \\
\bullet & \bullet \\
\bullet & \bullet \\
W_{1 j}^{(1)} & W_{2 j}^{(1)}
\end{array}\right] \cdot\left[\begin{array}{c}
F \\
V
\end{array}\right]\right)
$$

From (23), it can be simplified as:

$$
g(F, V)=f_{\text {Tanh }}\left(W^{(1)}(F, V)^{T}\right)
$$

The output of the neural network can be expressed as:

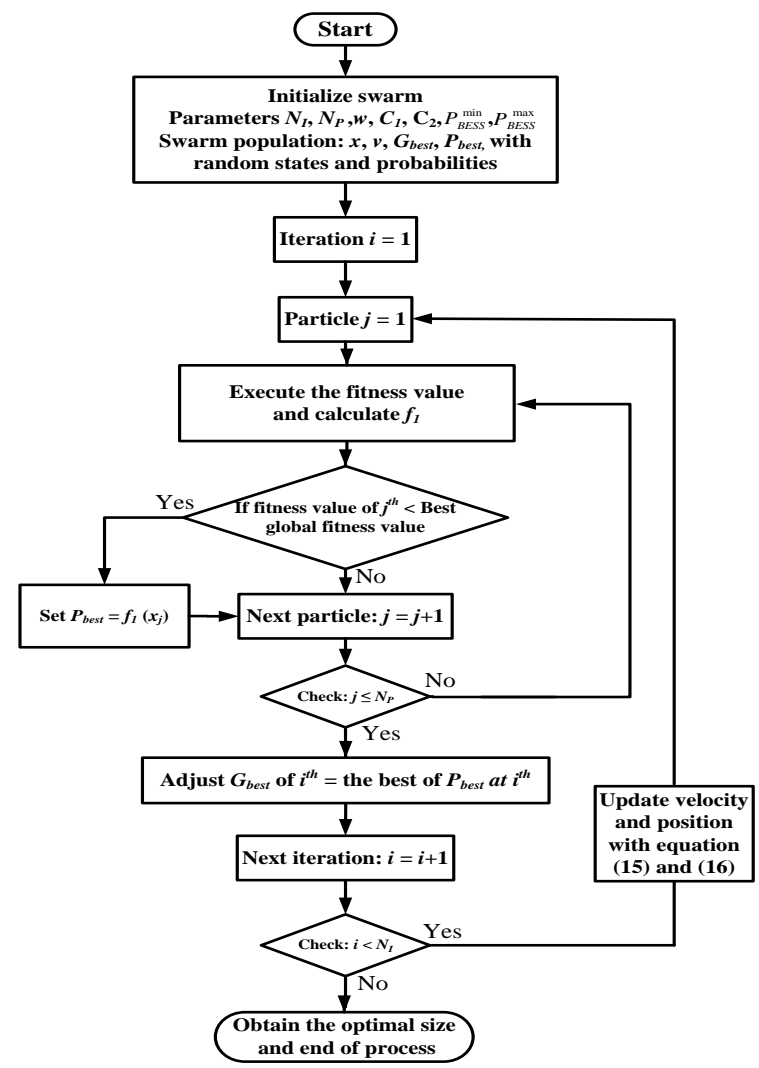

Fig. 5. Summarize of the optimal sizing of BESS-based PSO. 


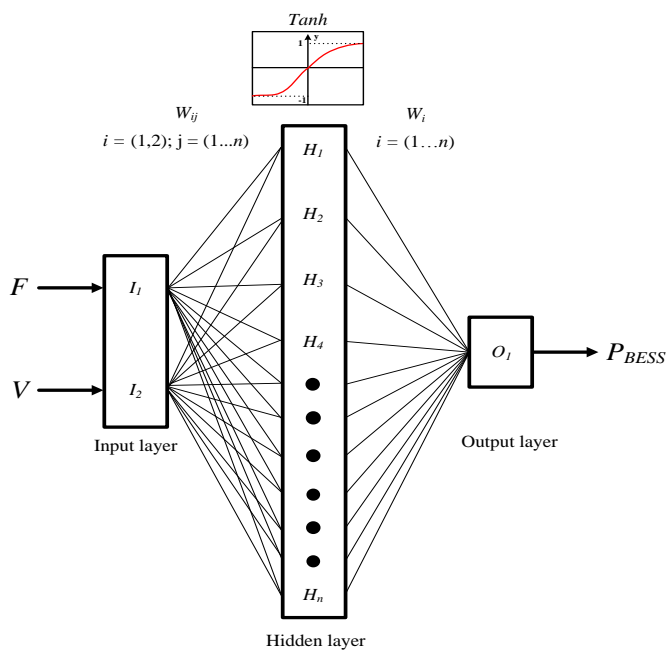

Fig. 6. Three layers architecture of the proposed neural network for BESS size optimization.

$$
P_{B E S S}(F, V)=f_{\text {Tanh }}\left(W^{(2)} g(F, V)\right)
$$

Finally, the proposed neural network model can be expressed as:

$$
P_{B E S S}(F, V)=f_{\text {Tanh }}\left(W^{(2)} f_{\text {Tanh }}\left(W^{(1)}(F, V)^{T}\right)\right)
$$

From Table I, the parameter details for the proposed neural network are shown and used in the training and the testing database.

\begin{tabular}{cc} 
TABLE I: MULTILAYER PERCEPTRON ANN PARAMETERS \\
\hline \hline Parameters & Multilayer perceptron ANN \\
\hline Goal (MSE) & 0.001 \\
\hline Inputs & 2 \\
\hline Outputs & 1 \\
\hline Hidden layer & 1 \\
\hline Training data & 30 \\
\hline Testing data & 4 \\
\hline Hidden layer neurons & 10 \\
\hline Output layer neurons & 1 \\
\hline Transfer function & Tanh \\
\hline \hline
\end{tabular}

Based on Fig. 7, the outline of the whole process of the proposed neural network is demonstrated. It can be seen that the mean square error (MSE) is compared between desired and actual data where the network will stop when the error less than 0.0001 .

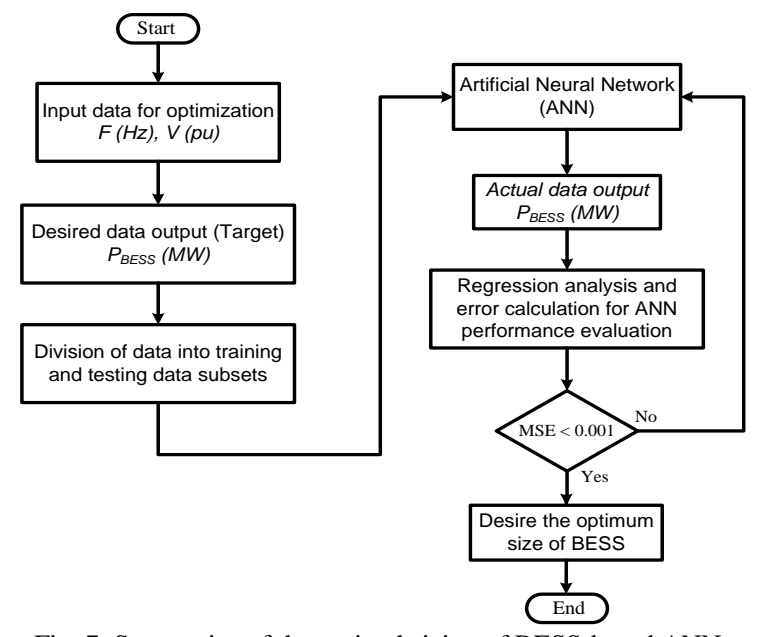

Fig. 7. Summarize of the optimal sizing of BESS-based ANN.

\section{Simulation Results}

This section illustrates the comparison results of the optimization techniques between Particle Swarm Optimization (PSO) and Artificial Neural Network (ANN) for evaluating the optimum size of BESS in the micro-grid system.

\section{A. PSO Results-Based BESS Size Optimization}

Fig. 8 and Table II, respectively, displays the convergence rate of the optimal sizing of BESS-based PSO and the optimized parameters such as the power capacity of BESS energy capacity of BESS, frequency and voltage of the micro-grid. Based on Fig. 8, the PSO algorithm is converged to its final state approximately after 15 iterations. It is evident that attainment is achieved with $G_{\text {best }}$ after $15^{\text {th }}$ iterations. The slightly attainment is reached with $G_{\text {best }}$ after $20^{\text {th }}$ iterations. However, no difference is observed after $20^{\text {th }}$ iterations. The reason for this is that this algorithm is symmetrical around the midpoint.

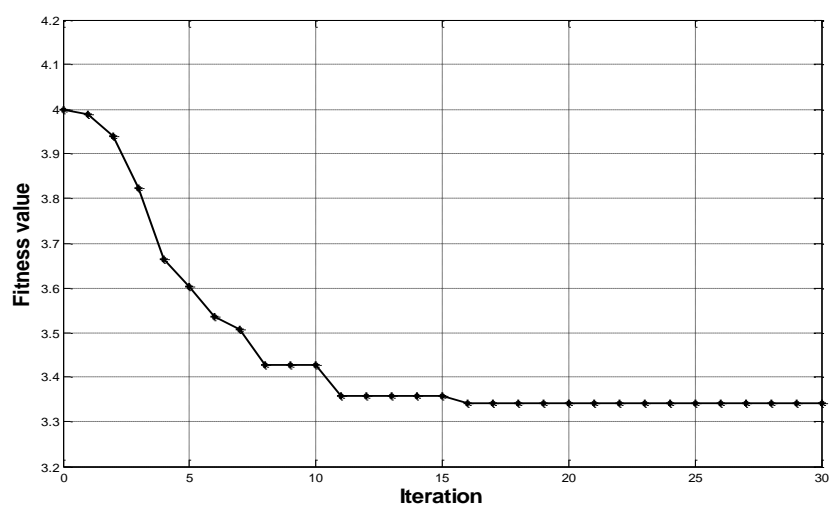

Fig. 8. Convergence rate of the optimal size of BESS-based PSO.

TABLE II: OPTIMIZED PARAMETER BASED ON PSO

\begin{tabular}{ccc}
\hline \hline$P_{\text {BESS }}(\mathrm{MW})$ & Frequency (Hz.) & Voltage (pu.) \\
\hline 3.3401 & 49.999 & 0.977 \\
\hline \hline
\end{tabular}

\section{B. ANN Results-Based BESS Size Optimization}

To demonstrate how well the proposed neural network model can achieve the nonlinear relationship between power capacity of BESS $\left(P_{B E S S}\right)$ and frequency and voltage of the micro-grid, the trained neural network is used to predict the power energy of BESS ( $\left.P_{B E S S}\right)$ by using the same input data as those in the training data set.

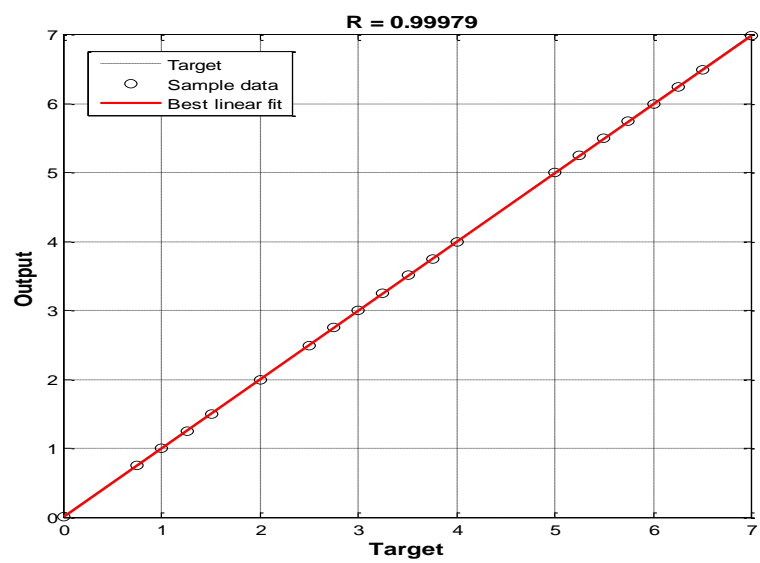

Fig. 9. Regression analysis between estimated output and measured target. 
In ANN training results, the outputs (the estimated $P_{B E S S}$ ) can be achieved accordingly. As shown in Fig. 9, the corresponding correlation coefficient $(\mathrm{R})$ is 0.99979 which is almost equal to 1 . Hence, the proposed neural network model is of high accuracy.

From ANN testing results, to illustrate the accuracy of the proposed neural network approach, the verification process is shown as Fig. 10. It can be seen that the estimated BESS size closely follows the pattern of measured BESS size. This confirms that the proposed neural network model is high accuracy.

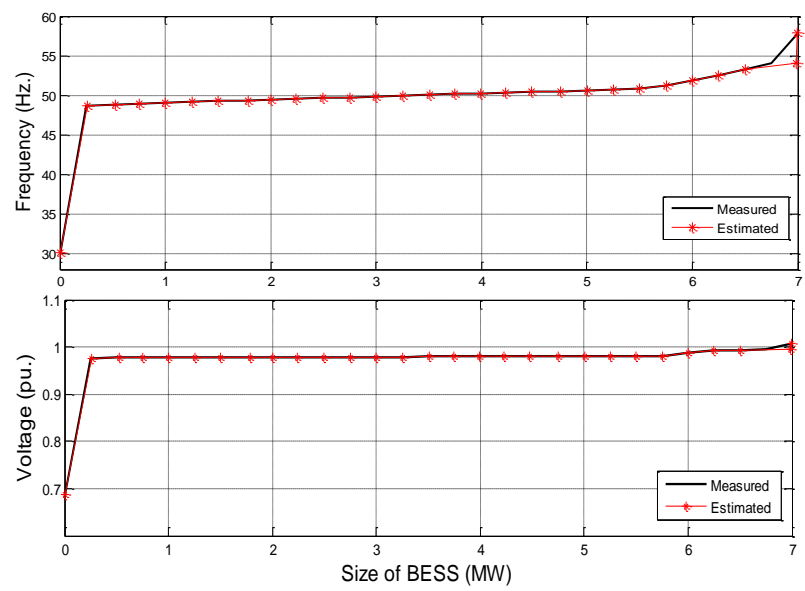

Fig. 10. Comparison between estimated BESS size and measured BESS size based on frequency and voltage of the micro-grid.

\begin{tabular}{cc}
\multicolumn{2}{c}{ TABLE III: MSE ERROR FOR BESS SIZE ESTIMATION } \\
\hline \hline Performance predictor & MSE \\
\hline$P_{\text {BESS }}$ & 0.00045623 \\
\hline \hline
\end{tabular}

The mean square error (MSE) on predicting performance shows the learning and generalization error of the normalized valued of BESS size (See Table III). This value revealed that the proposed neural network were able to accurately predict the optimum BESS size. MSE equation can be expressed as:

$$
M S E=\sqrt{\sum_{1}^{n}\left(T_{i}-O_{i}\right)^{2}}
$$

where $T_{i}$ is the target vector, $O_{i}$ is the output vector and $n$ is the number of training data or data for each test in the testing data set.

Based on Table IV, the optimal size of BESS is determined by the proposed neural network model based on frequency and voltage of the micro-grid.

TABLE IV: OPTIMIZED PARAMETER BASED ON ANN

\begin{tabular}{ccc}
\hline \hline$P_{\text {BESS }}(\mathrm{MW})$ & Frequency (Hz.) & Voltage (pu.) \\
\hline 3.3488 & 50.000 & 0.977 \\
\hline \hline
\end{tabular}

\section{Performance Comparison}

Based on Fig. 11, the peak magnitude of the frequency deviation with the BESS-based predefined size reaches 50.3 $\mathrm{Hz}$ and it cannot not recover to the normal state equilibrium (i.e., $50 \mathrm{~Hz}$ ). In case of no BESS, the system frequency dropped drastically because the power supply cannot meet the load demand. It can be seen in that the performance of system frequency is much better when the optimal sizing of BESS-based PSO or ANN is applied. It is clearly seen that the frequency performance of the optimum size of BESS-based ANN nearly follows the same pattern of the optimum size of based-PSO in terms of peak deviation and settling time.

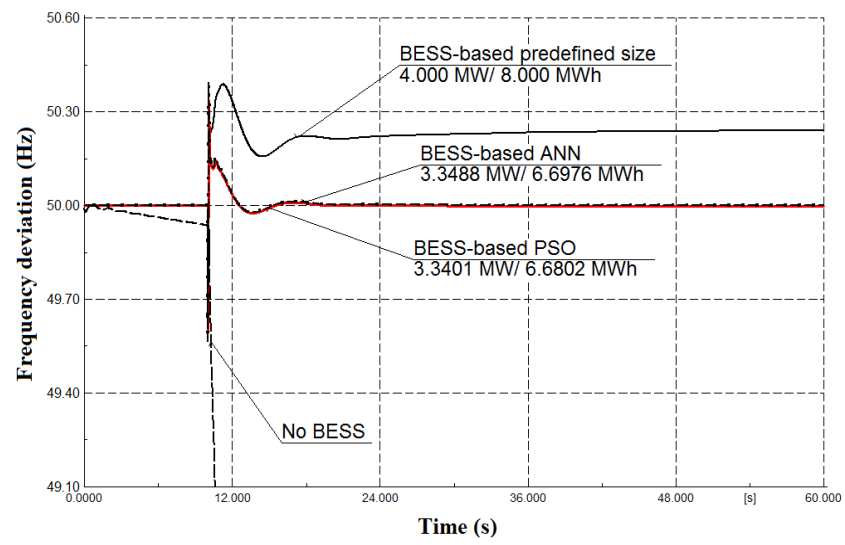

Fig. 11. Frequency deviation of the micro-grid after islanding.

Besides, the voltage deviation of the micro-grid system is shown in Fig. 12. According to this figure, the optimal sizing of BESS-based PSO/ANN can remain the same voltage deviation as the BESS-based predefined size. In particular, it is also seen that the voltage performance of the optimum size of BESS-based ANN nearly follows the same pattern of the optimum size of based-PSO in terms of peak deviation and settling time. In case of no BESS, the system voltage dropped drastically because the power supply cannot meet the load demand.

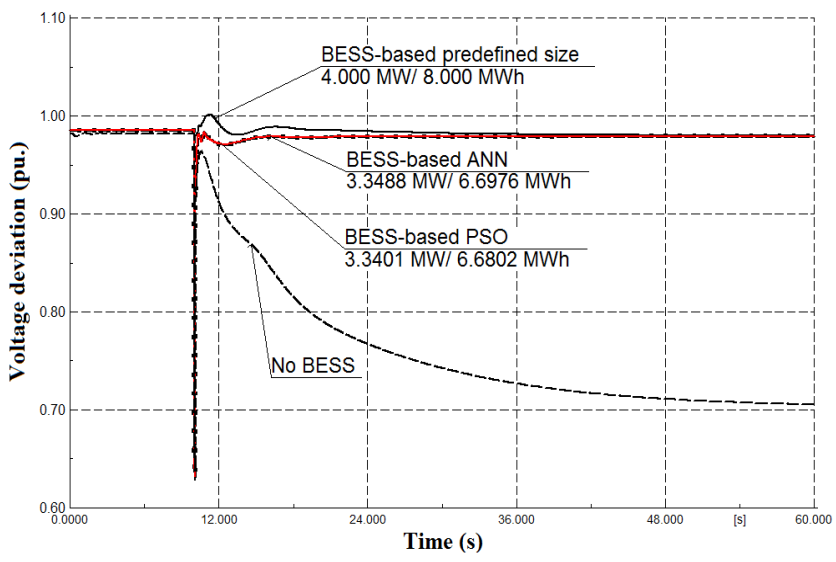

Fig. 12. Voltage deviation of the micro-grid after islanding.

From Table V, it can be observed that the optimum size of BESS-based ANN can closely obtain the same performance with the optimum BESS-based PSO. However, the optimal size of BESS-based PSO can achieve the lowest size of BESS while the performance of the optimal size of BESS-based PSO remains the same with the optimal size of BESS-based ANN. In particular, it should note that the accuracy of the performance of the optimum size of BESS-based ANN can be improved further by increasing the number of neurons and layers, but the time of calculation will be increased also. In terms of time of calculation, the optimum size of BESS-based ANN gives the best calculation time with only $11.0 \mathrm{~s}$ while the optimum size of BESS-based PSO gives the time of calculation with $285.0 \mathrm{~s}$. 
TABLE V: COMPARISON OF OPTIMIZED PARAMETERS

\begin{tabular}{ccccc}
\hline \hline Approach & $\begin{array}{c}P_{\text {BESS }} \\
(\mathrm{MW})\end{array}$ & $\begin{array}{c}\text { Frequency } \\
(\mathrm{Hz} .)\end{array}$ & $\begin{array}{c}\text { Voltage } \\
(\mathrm{pu} .)\end{array}$ & $\begin{array}{c}\text { Time of } \\
\text { calculation } \\
(\mathrm{sec} .)\end{array}$ \\
\hline PSO & 3.3401 & 49.999 & 0.977 & 285 \\
\hline ANN & 3.3488 & 50.000 & 0.977 & 11 \\
\hline \hline
\end{tabular}

\section{CONCLUSION}

This paper deals with the comparison of artificial intelligent approaches for determining the optimal BESS size in the micro-grid system. The BESS size is optimized by the artificial intelligence techniques which are PSO and ANN with feed forward feedback function. Results obtained from each approach are compared in terms of performance and time of calculation. As seen in the results, $3.3488-3.3401=$ $0.0087 \mathrm{MW}$ which means that the optimal size of BESS-based PSO can achieve the lowest size of BESS compared to the ANN-based optimization. While the performance of the two approaches remains the same, the calculation time significantly differ. The optimal size of BESS-based ANN shows shorter calculation time compared to the optimal size of BESS-based PSO (See Table V). Additionally, it can be conclude that the sizing of BESS-based PSO method can help to guarantee the lowest size of BESS with a reasonable and full use of the stand-alone micro-grid system, so that the micro-grid system can operate at the optimum conditions with optimal size of BESS in terms of investment and reliability requirement of the demand load.

\section{REFERENCES}

[1] J. Fossati, A. Galarza, A. Martin-Villate, J. Echeverria, and L. Fontan, "Optimal scheduling of a microgrid with a fuzzy logic controlled storage system," Int. J. Electr. Power. Energy. Syst., vol. 68, pp. 61-70, Jun. 2015.

[2] L. Prodan and E. Zio, "A model predictive control framework for reliable microgrid energy management," Int. J. Electr. Power. Energy. Syst., vol. 61, pp. 399-409, Oct. 2014.

[3] F. Katiraei, M. Iravani, and P. Lehn, "Micro-grid autonomous operation during and subsequent to islanding process," IEEE Trans Power Delivery, vol. 20, no. 1, pp. 248-257, Jan. 2005.

[4] J. Varela, L. J. Puglisi, T. Wiedemann, U. Ysberg, D. Stein, Z. Pokarna, C. Arnoult, R. Garaud-Verdier, and L. Consiglo, "Large-scale smart grid demonstrations for European distribution networks," IEEE Mag., Power \& Energy, vol. 13, no. 1, pp. 84-91, 2015.

[5] M. Koller, T. Borsche, A. Ulbig, and G. Andersson, "Review of grid applications with Zurich 1 MW battery energy storage system," Electrical Power Systems Research, vol. 120, pp. 128-135, 2015.

[6] M. Aghamohammadi and H. Abdolahinia, "A new approach for optimal sizing of battery energy storage system for primary frequency control of islanded microgrid," Int. J. Electr. Power. Energy. Syst., vol. 54, pp. 325-333, Jan. 2014.

[7] S. K. Aditya and D. Das, "Application of battery energy storage system to load frequency control of an islanded power system," Int. J. Energy Research, vol. 23, pp. 247-258, 1999

[8] T. Kerdphol, Y. Qudaih, and Y. Mitani, "Battery energy storage system size optimization in microgrid using particle swarm optimization," in Proc. IEEE PES Conf. on Innovative smart grid technologies (ISGT), 2014, pp. 1-6.

[9] F. Katiraei, M. Iravani, and P. Lehn, "Micro-grid autonomous operation during and subsequent to islanding process," IEEE Trans., Power Delivery, vol. 20, no. 1, pp. 248-257, Jan. 2005.

[10] J. Smith, R. Rylander, L. Rogers, and R. Dugan, "Maximizing the benefits and minimizing the impacts of DERs in an integrated grid," IEEE Power \& Energy Magazine, vol. 13, no. 2, pp. 20-29, 2015.

[11] K. Divya and J. Ostergaard, "Battery energy storage technology for power systems an overview," Electrical Power Energy Research, vol. 79, pp. 511-520, 2009
[12] G. Li, J. Na, D. Stoten, and X. Ren, "Adaptive neural network feedforward control for dynamically substructured systems," IEEE Trans. on Control System Tech., vol. 22, no. 3, pp. 944-954, 2014.

[13] H. A. Talebi and K. Khorasani, "A neural network-based multiplicative actuator fault detection and isolation of nonlinear system," IEEE Trans. on Control System Technology, vol. 21, no. 3, pp. 842-851, 2013.

[14] L. Shuai and L. Yangming, "Nonlinearity activated neural network for solving time-varying complex Sylvester equation," IEEE Trans. on Cybernetics, vol. 44, no. 8, pp. 1397-1407, 2014.

[15] W. Lin, C. Hong, C. Huang, and T. Ou, "Hybrid control of a wind induction generator based on grey-elman neural network," IEEE Trans. on Control System Technology, vol. 21, no. 6, pp. 2367-2373, 2013.

[16] G. Li, J. Na, D. P. Stoten, and X. Ren, "Adaptive neural network feedforward control for dynamically substructure systems," IEEE Trans. on Control System Tech., vol. 22, no. 3, pp. 944-954, 2014.

[17] J. Kennedy and R. Eberhart, "Particle swarm optimization," in Proc. IEEE Int. Conf. Neural Networks, 1995, vol. 4, pp. 1942-1948.

[18] P. Faria, Z. Vale, J. Soares, and J. Ferreira, "Demand response management in power systems using particle swarm optimization," IEEE Trans. on Intelligent Systems, vol. 28, no. 4, pp. 43-51, 2013.

[19] R. Cruz et al., "Particle swarm optimization for discrete-time inverse optimal control of a doubly fed induction generator," IEEE Trans. on Cybernetics, vol. 43, no. 6, pp. 1698-1709, 2013.

[20] R. Cheng and Y. Jin, "A competitive swarm optimizer for large scale optimization," IEEE Trans. on Cybernetics, vol. 45, no. 2, pp. 191-204.

[21] M. Alsayed, M. Cacciato, G. Scarcella, and G. Scelba, "Multicriteria optimal sizing of photovoltaic-wind turbine grid connected system," IEEE Trans. on Energy Conversion, vol. 28, no. 2, pp. 370-379, 2013.

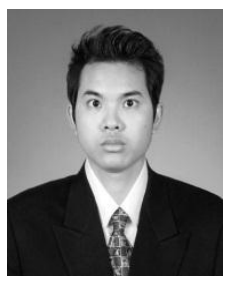

Thongchart Kerdphol received his B.Eng. and M.Eng degrees from Kasetsart University, Bangkok, Thailand in 2010 and 2012, respectively. Presently, he is pursuing a doctor of engineering degree sponsored by the Japan Student Services Organization (JASSO honors scholarship) at MITANI laboratory, Kyushu Institute of Technology (KIT). His research interest are in the area of the application of artificial intelligence in power systems, power system stability, smart/micro-grids, renewable energy, power system dynamics and controls. $\mathrm{He}$ is a member of IEEE.

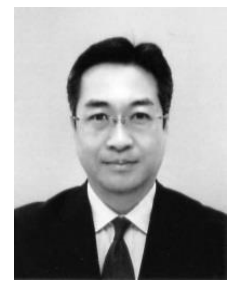

Kiyotaka Fuji received B.Sc., M.Sc. degrees in electrical engineering from Fukuoka University, Japan in 1992, 1994 respectively. He was with Yaskawa Electric Corporation, Japan from 1994 to 2011, and developed hydrogen fuel electric vehicle inverter, $\mathrm{PV}$ power conditioning system. He was with the Fellow Energy Co., Ltd. from 2012. At present, he is an adjunct lecturer at Kyushu Institute of Technology, Japan. His research interests are GIS power grid system, energy environment system, contactless power transfer, and $\mathrm{EV}$ power electronics devices. He is a member of IEEJ.

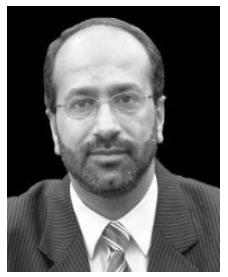

Yaser Soilman Qudaih graduated from the University of Engineering and Technology, Lahore, Pakistan in 1996 as an electrical engineer. $\mathrm{He}$ completed his M.Sc. and $\mathrm{PhD}$ degrees from Kumamoto University, Japan in electrical engineering. $\mathrm{He}$ is currently an assistant professor at the Department of Electrical Engineering and Electronics, Kyushu Institute of Technology (KIT), Japan. His area of interest including power system is renewable energy and smart grid applications. He is a member of IEEE.

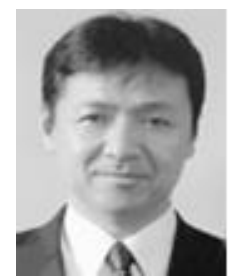

Yasunori Mitani received B.Sc., M.Sc. and D. Eng. degrees in electrical engineering from Osaka University, Japan in 1981, 1983 and 1986, respectively. He was a visiting research associate a the University of California, Berkeley, from 1994 to 1995. He is currently a professor at the Department of Electrical Engineering and Electronics, Kyushu Institute of Technology (KIT), Japan. His research interests are in the areas of analysis and control of power systems. He is a member of the Institute of Electrical Engineers of Japan and IEEE. 